\title{
Mecanismo de crescimento e caracterização de cristais formados na superfície de águas residuais de salinas
}

\author{
Liliane Ferreira Araújo de Almada ${ }^{1}$, Vitor Rodrigo Melo de Melo ${ }^{2}$, \\ Jussier de Oliveira Vitoriano ${ }^{2}$, Kristy Emanuel Silva Fontes ${ }^{1}$ \\ Francisco Edson Nogueira Fraga ${ }^{1}$, Clodomiro Alves Junior ${ }^{1,2}$
}

\footnotetext{
${ }^{1}$ Laboratório de Processamento de Materiais por plasma - Labplasma- DCEN/ UFERSA, Av. Francisco Mota, 572, CEP: 59.625-900, Mossoró, RN, Brasil.

${ }^{2}$ Programa de pós-graduação em Engenharia Mecânica, DEM-UFRN, Av. Sen. Salgado Filho, 3000, CEP: 59078-970, Natal, RN, Brasil.

e-mail: almadalily@gmail.com; clodomiro.jr@ufersa.edu.br
}

\section{RESUMO}

Sob condições ainda desconhecidas, cristais denominados de flor de sal, ou fleur de sel, como é comumente conhecido, pode ser formado na superfície de uma salmoura supersaturada durante a evaporação da água do mar nas salinas. Visando produzir flor de sal de uma maneira controlada e fazer melhor uso da solução residual de salinas, denominada de água-mãe, o presente trabalho foi proposto. O mecanismo de crescimento, morfologia, microestrutura e composição química dos sais obtidos foram estudados durante a evaporação da água-mãe, cuja densidade variou de $1,230 \mathrm{~g} / \mathrm{cm}^{3}$ para $1,244 \mathrm{~g} / \mathrm{cm}^{3}$, mantendo fixos o gradiente térmico e umidade relativa do ar. Análises quimicas, microestruturais e morfológicas foram usadas para caracterização. Cristais cúbicos ocos foram obtidos cujas concentrações iônicas foram dependents da densidade da solução. O tamanho e massa dos cristais também foram dependentes da densidade da solução. As amostras apresentaram fases contendo sulfatos como $\mathrm{NaK}_{3}\left(\mathrm{SO}_{4}\right)_{2}, \mathrm{Mg}\left(\mathrm{SO}_{4}\right)$. $\left(\mathrm{H}_{2} \mathrm{O}\right)$ e $\mathrm{K}_{2} \mathrm{SO}_{4}$, cuja porcentagem aumentou levemente com o aumento da densidade. Mesmo assim, as porcentagens de $\mathrm{NaCl}$ foram acima de 90\% para todas as amostras, mostrando que é possível extrair flor de sal de alta pureza a partir de uma solução residual hipersalina.

Palavras chaves: flor de sal, água-mãe, crescimento de cristais, mecanismo, salina.

\section{INTRODUÇÃO}

A produção de sal é geralmente obtido da captura da água do mar em reservatórios rasos onde a concentração dos íons é aumentada pela evaporação solar, até que o limite de solubilidade seja atingido, quando então o sal é precipitado [1-3]. Neste caso, a densidade da solução é um parâmetro muito importante para monitorar a evaporação natural durante a produção do sal. Quando a densidade da água do mar está entre 1,215 g/cm3 and 1,234 g/cm3, compostos de $\mathrm{NaCl}$ são precipitados [4]. Para densidade acima desse valor, a solução é descartada devido aos contaminantes como $\mathrm{Mg}$ e $\mathrm{K}$ presentes. Este produto, também denominado de águamãe, é rico em $\mathrm{Mg}^{2+}, \mathrm{Cl}^{-}, \mathrm{SO}_{4}{ }^{2-}, \mathrm{K}^{+}$, bem como íons de $\mathrm{Na}^{+}$. É uma forma concentrada de uma coleção de sais de magnésio, potássio, sulfato e cloreto, como $\mathrm{KCl}, \mathrm{MgCl} 2, \mathrm{Na} 2 \mathrm{SO} 4, \mathrm{MgSO} 4$ e sais duplos, como $\mathrm{K}_{\mathrm{x}} \mathrm{Na}_{(1-}$ ${ }_{x)} \mathrm{Cl}[5,6]$. A água-mãe não é apenas um resíduo da produção de sal, mas também de plantas de dessalinização por osmose reversa. Ela pode causar impactos ambientais devido à sua alta concentração de sais e outros conteúdos poluentes [7, 8]. ROBERT et al. [9] descrevem os impactos do descarte da água-mãe como impactos físico-químicos e ecológicos, nos quais o primeiro pode alterar as propriedades físicoquímicas da água receptora, afetando a vida marinha de várias maneiras e o segundo pode prejudicar os organismos aquáticos, uma vez que a solução pode conter componentes tóxicos. Indústrias têm se concentrado na redução e nas opções de reutilização das águas-mães. Além disso, esse efluente pode ser considerado uma fonte potencial para usos valiosos permitindo ainda reduções de custos [7, 10]. Nos processos de dessalinização, a solução rejeitada pode ser tratada com dupla finalidade, de recuperação da água e produção de sal simultaneamente $[11,12]$.

Um fenômeno muito interessante durante a produção de sal é a ocorrência de cristais na superfície da 
salmoura, além dos sais precipitados e sedimentados no fundo dos reservatórios das Salinas. Estes cristais são formados sob condições específicas de temperatura, umidade e vento [13, 14]. Eles receberam inicialmente o nome de fleur de sel, porque a frança foi o primeiro país a explorá-lo comercialmente. Ele é conhecido como o "caviar do sal" devido, porque possui sabor singular e alto preço no mercado [13, 15]. Sua coleta é artesanal, mas não necessita de qualquer processamento adicional, sendo diretamente embalado e comercializado. Sua produção não é controlada devido sua dependência com fatores climáticos, pois fortes ventos e alta umidade torna impossível sua produção $[15,16]$. Num raro e clássico estudo sobre o tema, DELLWIG [17], afirma que esses cristais forma quando a temperatura de evaporação está abaixo da ebulição, permitindo a formação do filme fino de alta densidade na superfície da salmoura, propiciando a nucleação e cristalização. Na medida que o crescimento continua, os cristais tendem a afundar devido ao seu próprio peso, mas são sustentados pela tensão superficial. Como apenas uma face do cubo está em contato com o filme fino de alta densidade, o crescimento ao longo de suas bordas é maior do que as partes que não possuem contato com o filme, resultando em uma pirâmide oca com o ápice voltado para baixo [18, 19]. Qualquer perturbação da superfície pode levar à inundação e sedimentação dos cristais, sendo o seu manuseio extremamente delicado. Neste trabalho foi estudada a cristalização de sais na superfície da água-mãe. Atualmente, não existem dados que expliquem a influência de qualquer um dos fatores ambientais envolvidos no processo de formação e crescimento desses cristais, bem como sua morfologia e composição química. Partimos da hipótese da influência de um gradiente térmico formado entre a solução e o ar ambiente, para estudar a cinética da formação e crescimento de cristais na superfície da água-mãe com base na morfologia, composição química e microestrutura em comparação com a flor de sal comercial.

\section{MATERIAIS E MÉTODOS}

Para a simulação experimental, $150 \mathrm{~mL}$ de uma solução salina hiperconcentrada, água mãe, foram acondicionados em um recipiente cilíndrico de alumínio, $80 \mathrm{~mm}$ de diâmetro, contendo um isolamento externo em isopor. Para a obtenção dos cristais na superfície, o recipiente contendo a solução foi colocado sobre uma chapa de aquecimento a temperatura fixa de $40{ }^{\circ} \mathrm{C}$ por 18 horas, enquanto a temperatura ambiente do laboratório permaneceu constante em $25{ }^{\circ} \mathrm{C}$. A umidade relativa do ar foi mantida em $50 \%$. Três termopares foram utilizados em diferentes posições para capturar variações de temperatura na superfície da solução $\left(\mathrm{T}_{\text {sup }}\right), 10 \mathrm{~mm}$ acima $\left(\mathrm{T}_{\text {up }}\right)$ e $10 \mathrm{~mm}$ abaixo $\left(\mathrm{T}_{\text {down }}\right)$. Durante o experimento, vídeos da superfície da solução foram gravados com o auxílio de uma lupa estereoscópica Nikon, modelo MSZ 18, acoplada também a uma câmera Nikon, modelo DS-F12. Na figura 1 é mostrado o esquema correspondente ao aparato experimental.

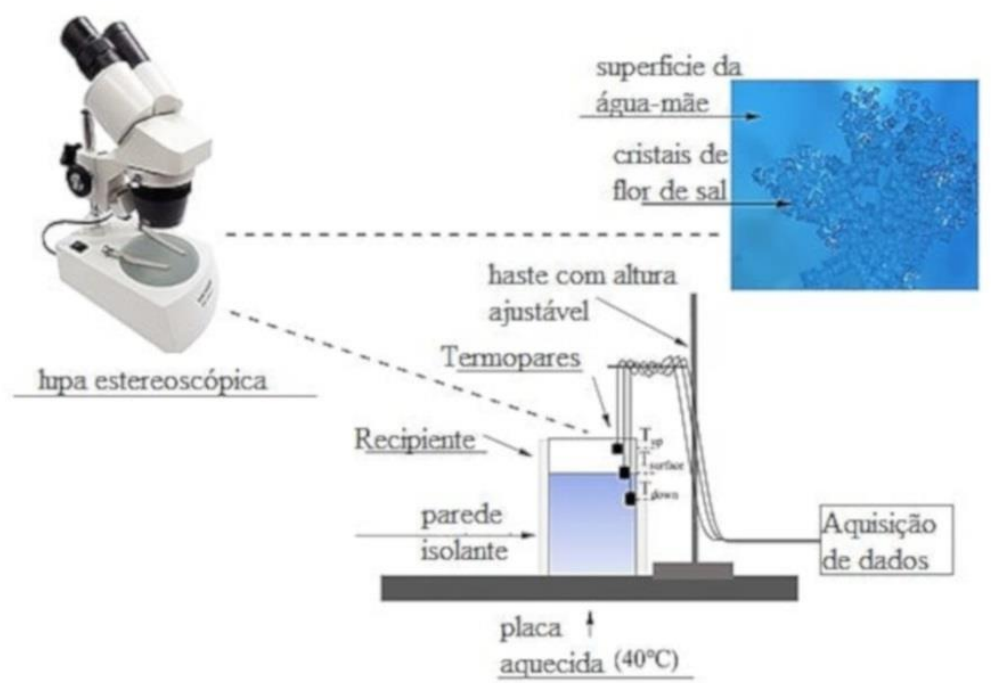

Figura 1: Aparato experimental para monitoramento do crescimento dos cristais.

Após as 10 primeiras horas, em temperatura constante, os cristais formados na superfície foram coletados de 2 em 2 horas, totalizando 5 amostras de flor de sal. Durante cada coleta, foram realizadas medições da densidade da solução, da umidade relativa do ar com o auxílio de um termohigrômetro KASVI (modelo K29-5070H) e a retirada de pequenas alíquotas da solução para a realização das análises químicas. As amostras coletadas foram aquecidas a $60^{\circ} \mathrm{C}$ para secagem em estufa. 


\subsection{Análises químicas}

As amostras de flor de sal e as alíquotas da solução, coletadas simultaneamente, foram analisadas quimicamente para determinar as concentrações percentuais dos íons $\mathrm{Na}^{+}, \mathrm{K}^{+}, \mathrm{Ca}^{2+}, \mathrm{Mg}^{2+}, \mathrm{Cl}^{-}$e $\mathrm{SO}_{4}^{2-}$. Para a determinação dos íons na flor de sal, $20 \mathrm{mg}$ foram dissolvidos em $50 \mathrm{~mL}$ de água deionizada. As concentrações iônicas de sódio, cálcio e potássio foram determinadas por espectrofotometria em um fotômetro de chama Digimed, modelo DM-62, utilizando soluções analíticas de 2, 4, 6, 8 e 10 ppm dos respectivos íons. A concentração do íon sulfato foi determinada por espectroscopia de absorção óptica, utilizando o método turbidimétrico em um espectrofotômetro UV-VIS, modelo Genesys 10S. As soluções analíticas continham 10,20,30,50, 80 e 120 ppm de enxofre, com faixa de medição de 410 a $420 \mathrm{~nm}$. Os íons $\mathrm{Cl}^{-}$e $\mathrm{Mg}^{+}$foram determinados pelo método de Mohr e por titulação complexométrica, respectivamente. O nitrato de prata, titulante utilizado na determinação de cloro, possuía concentração de $0,01 \mathrm{M}$ para a análise das amostras de flores de sal e 0,1M para as alíquotas da solução. As concentrações iônicas de $\mathrm{Mg}^{2+}$ foram determinadas pela diferença entre a dureza da água e a concentração de íons $\mathrm{Ca}^{2+}$. O titulante para determinação da dureza consistiu em uma solução de EDTA 0,001M e 0,1M para as amostras de flores de sal e solução, respectivamente. Todas as análises foram realizadas em triplicata.

\subsection{Análise microestrutural}

A morfologia dos cristais foi analisada a partir de imagens obtidas após secagem em lupa estereoscópica Nikon MSZ 18 acoplada a uma câmera Nikon DS-F12 e por microscopia eletrônica de varredura (MEV) em um Tescan Vega3, equipado com um filamento de tungstênio operando a $20 \mathrm{kV}$. Os tamanhos dos cristais foram determinados por medições utilizando um software de análise de imagem, Image J. As estruturas das amostras foram caracterizadas por difração de raios X (DRX), utilizando um difratômetro da Shimadzu, modelo XRD 6000, operando a $40 \mathrm{kV}$ e $30 \mathrm{~mA}$, equipado com um tubo de radiação $\mathrm{CuK} \alpha(\lambda=1,5409 \AA)$. A identificação das fases cristalinas foi realizada por meio do software Highscore Plus, da PANalytical, com base no banco de dados do ICSD (Inorganic Crystal Structure Database) e COD (Crystallography Open Database). O refinamento da estrutura foi realizado no software Maud (Material Analysis Using Diffraction), aplicando o tratamento Rietveld.

\section{RESULTS}

\subsection{Cinética de crescimento e morfologia dos cristais}

Nos primeiros minutos, durante a etapa de aquecimento, microbolhas de ar são observadas na solução (Fig. 2.a). As microbolhas movimentam-se para a parte superior da solução devido as correntes de convecção decorrentes das trocas térmicas. Nesse momento, os íons solvatados estão espalhados uniformemente no líquido. Após 2 horas de aquecimento, ocorre o surgimento dos primeiros cristais na superfície (Fig. 2.b). Uma explicação para esse fenômeno foi apresentado na literatura considerando a supersaturação local na interface da solução salina [17]. Em nosso experimento foi fixado uma diferença de temperatura entre a superfície da água e o ambiente $\left(\mathrm{T}_{\text {sup }}-\mathrm{T}_{\text {up }}\right)$ de $5,3^{\circ} \mathrm{C}$ e de $2,3{ }^{\circ} \mathrm{C}$ para $\left(\mathrm{T}_{\text {down }}-\mathrm{T}_{\text {sup }}\right)$. Essa diferença no gradiente térmico pode favorecer uma maior mobilidade das partículas na superfície da solução que no interior, aumentando assim a seção de choque dos íons solvatados e consequente precipitação dos sais. Acontece que, para os cristais crescerem, é necessário núcleos que atuem como centros de cristalização. As microbolhas provavelmente tiveram a função de sítios de nucleação. Verificou-se que a densidade de bolhas era reduzida na medida que os cristais começavam a aparecer. Em constante formação, os pequenos cristais passam a crescer e se aglutinar em posições aleatórias, formando, após as 10 primeiras horas de iniciado o processo de aquecimento, uma espécie de nata na superfície que permanece sustentada pela tensão superficial até que o peso se torne maior que a força de sustentação e a placa cristalina decante no fundo do recipiente (Fig. 2.c). Outro fator de sustentação desta placa está relacionado com a presença de vazios observados no interior dos cristais cúbicos (Fig. 2.d). Além da supersaturação, acredita-se que as microbolhas de ar presentes na solução tenham aderido à primeira camada formada do cristal e as demais tenham surgido em volta dela, resultando na presença dos vazios $[20,21]$. 

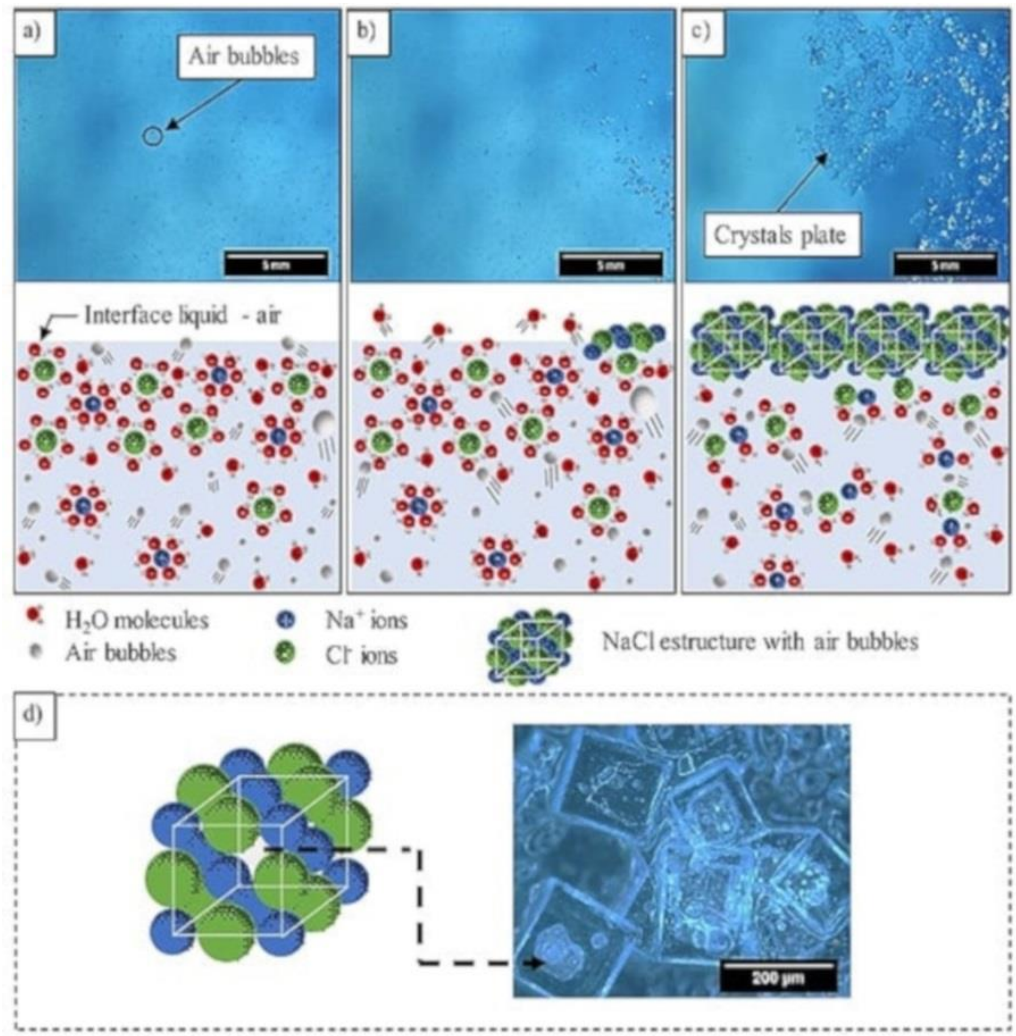

Figura 2: Imagem obtida por lupa estereoscópica durante a evaporação da água-mãe, mostrando a evolução do crescimento dos cristais a) após $30 \mathrm{~min} \mathrm{~b}$ ) após $120 \mathrm{~min}$ and c) após $600 \mathrm{~min}$; d) imagem do cristal ilustrando bolhas de ar na coleta de número 5. Abaixo das imagens a, b e c, modelo hipotético, em nível molecular, das respectivas etapas.

A morfologia das amostras de flor de sal apresentadas na Figura 3, revela um material formado por cristais aglomerados aleatoriamente com estruturas cúbicas características do $\mathrm{NaCl}$. A aglomeração, que ocorre pela coalescência entre os cristais, aumenta da primeira para a última coleta e a estrutura cúbica fica menos evidente devido a sobreposição das estruturas cúbicas individuais. A partir das micrografias dos cristais obtidos, foi possível estimar, utilizando o software ImageJ, o tamanho médio dos cristais em cada coleta. As curvas do tamanho médio dos cristais, da massa total de flor de sal e da densidade da solução no decorrer do tempo de evaporação são também apresentadas na Figura 3.

O tamanho do cristal variou inversamente com a progressão do tempo de evaporação. Entretanto, um resultado aparentemente conflitante está na diminuição da massa total dos cristais. Como o tamanho menor de cristais está associado com o aumento no número de sítios de nucleação, se esperava que também a massa total aumentasse. O aumento na densidade da solução com a evaporação é outra razão para afirmar que o número de sítios de nucleação aumentou, devido à maior saturação da solução [22, 23]. A única explicação plausível para a massa total menor desses cristais é a maior presença de vazios nos cristais posteriores à $3^{\mathrm{a}}$ coleta.

A variação da densidade da solução está diretamente relacionada com o aumento na concentração decorrente da evaporação. A concentração iônica é alterada positivamente pela evaporação e negativamente pelas precipitações que ocorrem quando o limite de saturação para um determinado sal é atingido [24]. Com base nessas observações, nossa hipótese é que a formação de cristais flutuantes ocorra devido à evaporação mais intensa e maior mobilidade dos íons solvatados, que promove a precipitação local. Esses precipitados crescem na interface bolha-água, que atuam como sítios de nucleação. A morfologia cúbica, com vazios internos no filme cristalino formado, permite aumentar a força de empuxo do líquido, mantendo-o flutuante. 

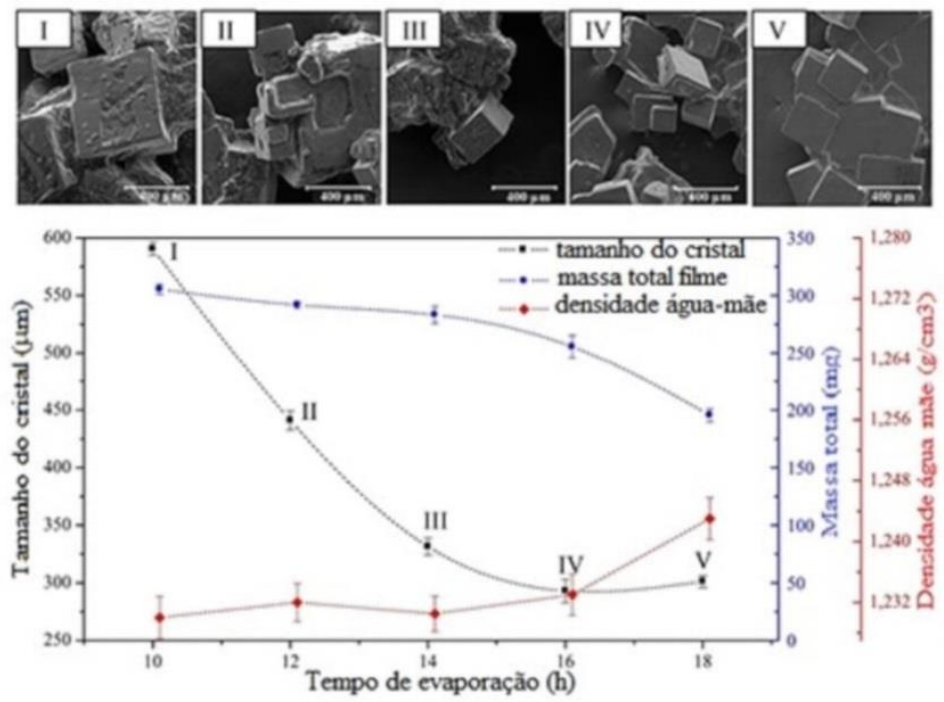

Figure 3: Evolução do tamanho e massa total dos cristais para diferentes densidades da água-mãe obtidas quando a evaporação progride com o tempo. Na parte superior, as micrografias dos cristais obtidos nas cinco coletas.

\subsection{Análise química}

Na Figura 4, estão apresentadas as concentrações dos íons presentes na solução inicial e nas alíquotas extraídas em cada coleta. Tanto na solução inicial como nas alíquotas subsequentes, foram observados grandes percentuais de $\mathrm{Cl}^{-}, \mathrm{Na}^{+}$e $\mathrm{Mg}^{2+}$ em relação aos demais íons constituintes. No decorrer das coletas, com o aumento na densidade da solução, foi observada uma pequena variação positiva nos percentuais de $\mathrm{K}^{+}$, $\mathrm{Mg}^{2+}, \mathrm{SO}_{4}{ }^{2-}$. $\mathrm{O}$ aumento no percentual desses íons está relacionado com o decréscimo na concentração do $\mathrm{Na}^{+}$na solução, devido ao aumento sistemático de sódio em cada coleta de flor de sal (Fig. 5). Como esperado, grandes percentuais de cloro, sódio e magnésio foram encontrados na composição da água mãe com densidade inicial de $1,23 \mathrm{~g} / \mathrm{cm}^{3}$. Mesmo com altos valores de concentração de sódio e cloro, essa solução não produz $\mathrm{NaCl}$ com alta pureza devido, principalmente, à presença de magnésio [4]. Observa-se pela figura 4 que, de um modo geral, há um decréscimo nas concentrações de $\mathrm{Na}, \mathrm{Cl}$ na medida que progride a evaporação, enquanto as concentrações de $\mathrm{Mg}, \mathrm{K} \mathrm{e} \mathrm{SO}_{4}$ são aumentadas. Exceção apenas para a $4^{\mathrm{a}}$ coleta, na qual ocorre um decréscimo na concentração de $\mathrm{K}$ e $\mathrm{SO}_{4}$. $\mathrm{O}$ decréscimo nas concentrações pode ser atribuído à precipitação na flor de sal ou nos precipitados sedimentados no fundo do recipiente.

$\mathrm{Na}$ Figura 5, estão apresentadas as concentrações percentuais dos íons presentes nas amostras de flor de sal coletadas. Como pode ser visto, as concentrações de $\mathrm{Na}$ e $\mathrm{SO}_{4}$ aumentaram sistematicamente com o tempo de evaporação, enquanto a de cloro foi reduzida. Por outro lado, as concentrações de $\mathrm{K}$ e $\mathrm{Mg}$ se mantiveram praticamente constantes. Este é um resultado interessante porque mostra que a flor de sal está sendo mais seletiva na extração de sódio que potássio. 


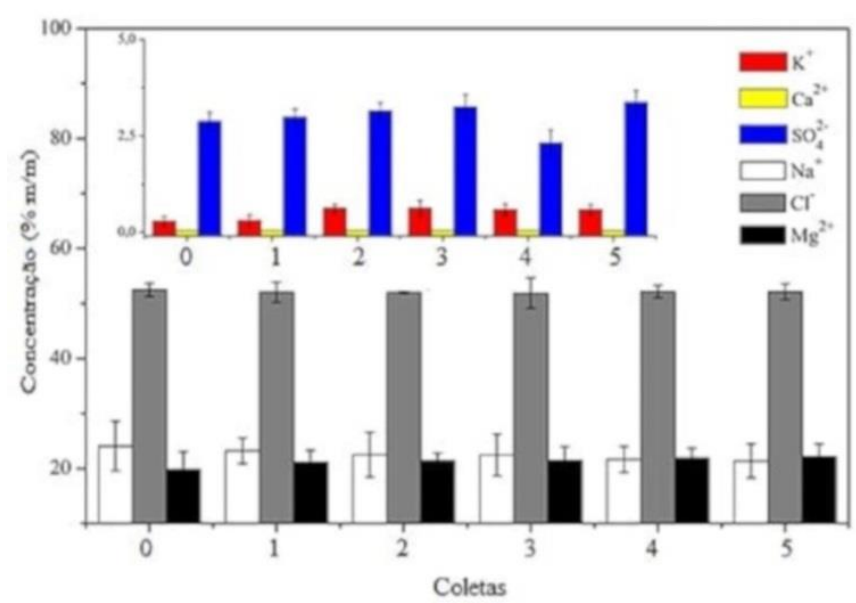

Figure 4: Concentração de íons presentes na água-mãe no momento de cada coleta. Gráfico acima mostra os componentes minoritários.

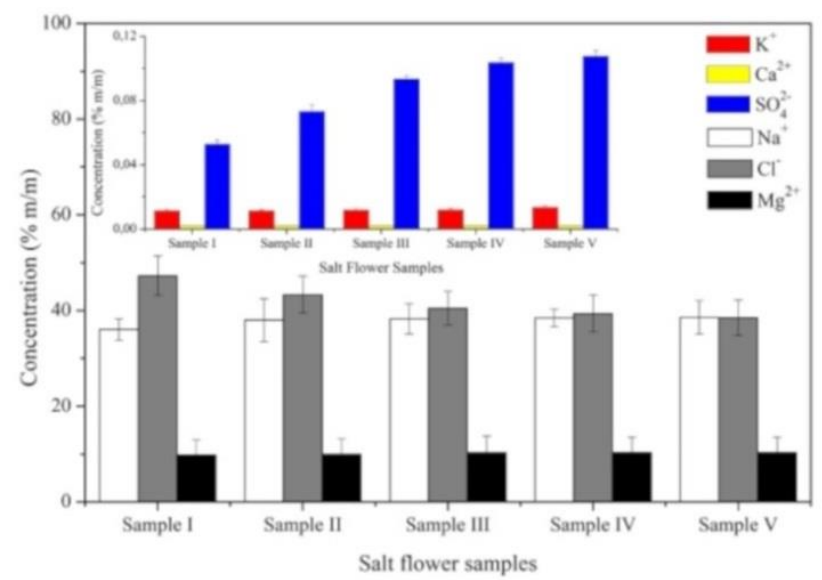

Figure 5: Concentração de íons presente em cada amostra de flor de sal coletada.

Os valores observados nas coletas foram comparados com os valores obtidos a partir da solução inicial e estão representados na Tabela 1. Considerando as porcentagens de $\mathrm{Cl}^{-}$e $\mathrm{Na}^{+}$na flor de sal e a facilidade da ligação entre eles, pode-se considerar que são estequiometricamente suficientes para formar $\mathrm{NaCl}$ em grande quantidade. Sendo assim, os cristais formados devem ser compostos de percentuais entre 90 e $95 \%$ de $\mathrm{NaCl}$. $\mathrm{O}$ aumento da concentração de sódio e de $\mathrm{SO}_{4}$, concomitante à redução de cloro, indica uma possibilidade de precipitação de sulfatos em substituição aos cloretos. Conforme esses íons vão sendo removidos da solução

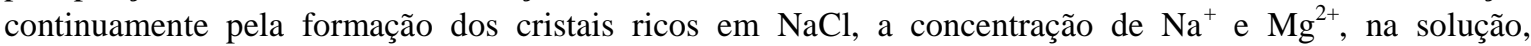
praticamente competem entre si, de modo que, a partir de 18 horas de evaporação, densidade correspondente a 1,244 , a porcentagem de $\mathrm{Mg}^{2+}$ se torna levemente superior à de $\mathrm{Na}^{+}$. Esse resultado mostra que a supersaturação na superfície ocorre preferencialmente para formar o $\mathrm{NaCl}$, invés de $\mathrm{MgSO}_{4}$ ou $\mathrm{MgCl}_{2}$, até mesmo quando a solução apresenta maior porcentagem de íons magnésio e cloro em relação ao sódio. Podemos concluir desses resultados, que é possível produzir flor de sal a partir da água residual da salina e que durante sua produção há extração seletiva do $\mathrm{Na}^{+} \mathrm{em} \mathrm{vez} \mathrm{do} \mathrm{Mg}^{2+}$, resultando num produto com alta pureza (maior que $90 \%$ de $\mathrm{NaCl}$ ). Também essa metodologia pode ser interessante como rota de separação da água-mãe. 
Table 1. Concentração de íons presentes nas amostras de flor de sal e da solução de água-mãe para as diferentes coletas (média de 3 repetições)

\begin{tabular}{|c|c|c|c|c|c|c|}
\hline $\begin{array}{c}\text { Concentration } \pm \\
\text { desviation }(\% \\
\mathbf{m} / \mathbf{m})\end{array}$ & $\mathrm{Na}^{+}$ & $\mathrm{Cl}^{-}$ & $\mathbf{K}^{+}$ & $\mathrm{Ca}^{2+}$ & $\mathrm{SO}_{4}{ }^{2-}$ & $\mathrm{Mg}^{2+}$ \\
\hline \multicolumn{7}{|c|}{ Flor de sal } \\
\hline Sample I & $36,05 \pm 2,21$ & $47,27 \pm 4,12$ & $1,14 \pm 0,08$ & $0,19 \pm 0,00$ & $5,29 \pm 0,26$ & $9,82 \pm 3,12$ \\
\hline Sample II & $38,01 \pm 4,51$ & $43,33 \pm 4,33$ & $1,14 \pm 0,09$ & $0,19 \pm 0,00$ & $7,32 \pm 0,40$ & $9,93 \pm 3,21$ \\
\hline Sample III & $38,27 \pm 3,13$ & $40,48 \pm 4,04$ & $1,19 \pm 0,09$ & $0,19 \pm 0,00$ & $9,35 \pm 0,20$ & $10,26 \pm 3,49$ \\
\hline Sample IV & $38,43 \pm 1,85$ & $39,39 \pm 3,86$ & $1,20 \pm 0,09$ & $0,19 \pm 0,00$ & $10,37 \pm 0,26$ & $10,32 \pm 3,12$ \\
\hline Sample V & $38,58 \pm 3,49$ & $38,49 \pm 3,68$ & $1,36 \pm 0,09$ & $0,19 \pm 0,00$ & $10,77 \pm 0,36$ & $10,32 \pm 3.12$ \\
\hline \multicolumn{7}{|c|}{ Água-mãe } \\
\hline Initial solution & $24,06 \pm 4,50$ & $52,49 \pm 1,18$ & $0,29 \pm 0,12$ & $0,05 \pm 0,00$ & $2,88 \pm 0,24$ & $19,82 \pm 3,20$ \\
\hline Aliquot 1 & $23,17 \pm 2,36$ & $52,03 \pm 1,84$ & $0,31 \pm 0,14$ & $0,05 \pm 0,00$ & $2,99 \pm 0,21$ & $21,14 \pm 2,10$ \\
\hline Aliquot 2 & $22,49 \pm 4,09$ & $51,98 \pm 0,24$ & $0,62 \pm 0,11$ & $0,05 \pm 0,00$ & $3,15 \pm 0,22$ & $21,39 \pm 1,40$ \\
\hline Aliquot 3 & $22,42 \pm 3,80$ & $51,86 \pm 2,75$ & $0,62 \pm 0,21$ & $0,05 \pm 0,00$ & $3,26 \pm 0,31$ & $21,46 \pm 2,50$ \\
\hline Aliquot 4 & $21,64 \pm 2,36$ & $52,16 \pm 1,12$ & $0,59 \pm 0,15$ & $0,05 \pm 0,00$ & $2,32 \pm 0,34$ & $21,90 \pm 1,80$ \\
\hline Aliquot 5 & $21,35 \pm 3,10$ & $52,13 \pm 1,43$ & $0,59 \pm 0,13$ & $0,04 \pm 0,00$ & $3,37 \pm 0,31$ & $22,15 \pm 2,30$ \\
\hline
\end{tabular}

\subsection{Análise quantitative das fases cristalinas}

Os padrões de DRX das amostras coletadas são apresentados na Figura 6. Todas as amostras apresentam picos de difração característicos da estrutura cúbica da halita ( $2 \theta$ em 31-32 e 45-46 $)$, bem como a presença de picos característicos de sais duplos do tipo $\mathrm{Na}_{\mathrm{x}} \mathrm{K}_{1-\mathrm{x}} \mathrm{Cl}$ também com estrutura cúbica $\left(2 \theta\right.$ em $30,2^{\circ}$ e $43,2^{\circ}$, principalmente) de acordo com os códigos das cartas cristalográficas apresentados na Tabela 2. Entretanto, fases de sulfatos $\left(\mathrm{M}_{\mathrm{y}} \mathrm{SO}_{4}\right.$, onde $\mathrm{M}_{\mathrm{y}}=\mathrm{Na}, \mathrm{K}$ ou $\left.\mathrm{Mg}\right)$, com estruturas monoclínicas, também foram identificadas e suas concentrações são dependentes da densidade. Quanto mais densa a solução maior é a presença de sulfatos nas amostras de flor de sal. Outro dado importante é que a intensidade relativa do pico (020) é sempre maior que o valor existente nas cartas cristalográficas. Isso é uma forte indicação que houve crescimento preferencial na direção $\langle 020\rangle$. O comparativo entre os difratogramas das amostras extraídas a partir de soluções com diferentes densidades e o difratograma de uma amostra de flor de sal comercial nos mostra que, nas três primeiras coletas, existe maior porcentagem de $\mathrm{NaCl}$, indicando um alto grau de pureza das amostras obtidas.

O cálculo da razão percentual das fases cristalinas conforme mostrado pelo refinamento Rietveld (Tabela 2), apresenta uma correlação da redução da porcentagem de $\mathrm{NaCl}$ com o aumento da densidade, variando de $94,92 \%$ na primeira coleta para $90,54 \%$ na última. Com o decréscimo do $\mathrm{NaCl}$ no decorrer das coletas, é observado um aumento na porcentagem de fases de sulfatos, que vai de um total de 3,60\% na amostra I para $6,71 \%$ na amostra $\mathrm{V}$, confirmando o aumento do íon sulfato na análise química (Tabela 1).

Comparando os difratogramas das amostras obtidas com uma amostra de flor de sal comercial, pode-se observar que, apesar das diferentes fases que surgem com o aumento da densidade, as amostras apresentam porcentagens de $\mathrm{NaCl}$ e cristalinidade elevadas (Tabela 2), sugerindo um alto grau de pureza, assim como observado na flor de sal comercial. 

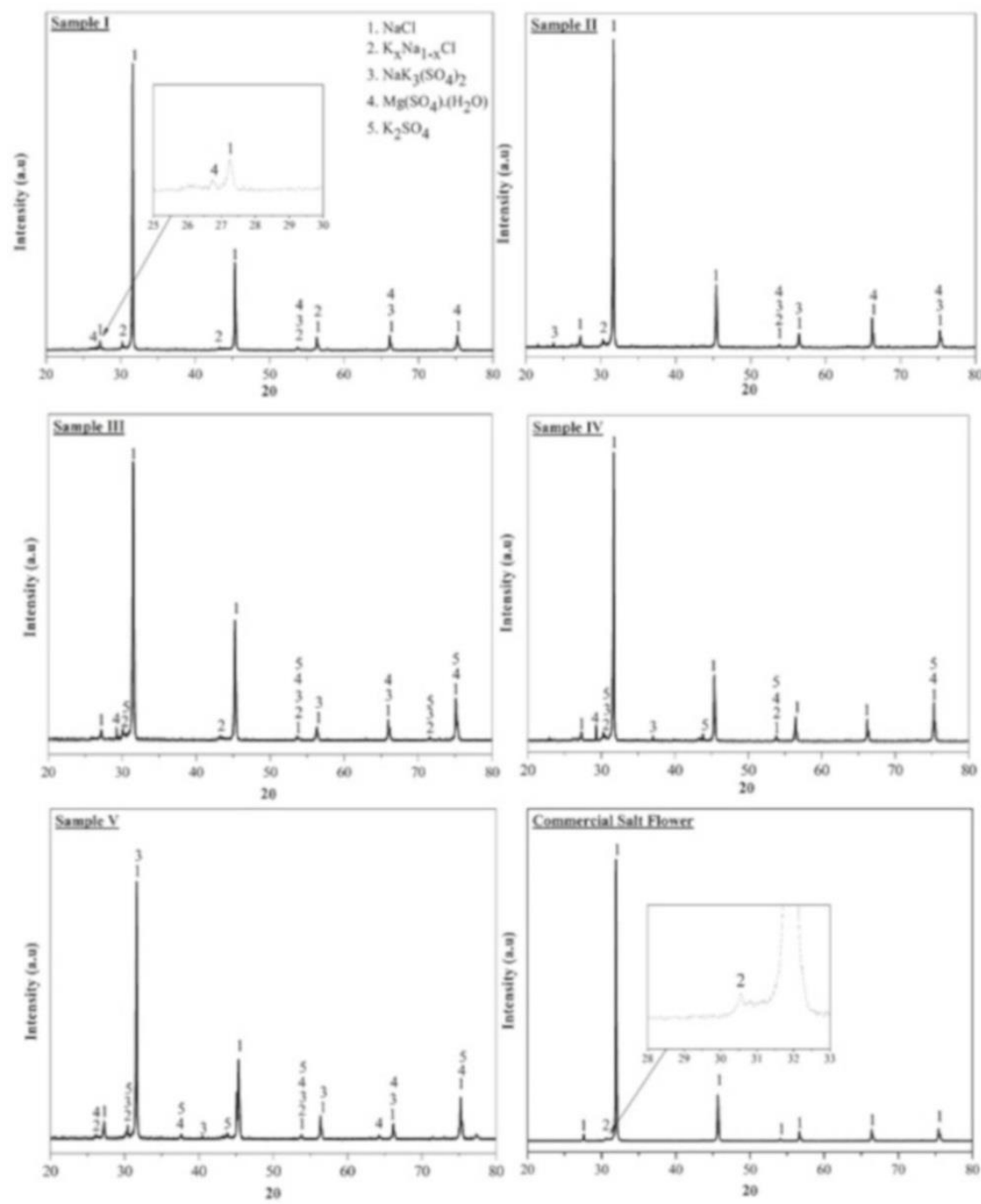

Figura 6: Difratogramas de flor de sal das cinco coletas realizadas durante a evaporação da água-mãe e de amostra de flor de sal comercial.

Table 2: Concentração, cristalinidade e parâmetros de refinamento das fases cristalinas das amostras de flor de sal.

\begin{tabular}{|c|c|c|c|c|c|c|c|c|}
\hline \multirow[t]{2}{*}{ Amostra } & \multicolumn{5}{|c|}{ Fases $(\% p)^{a}$} & \multirow{2}{*}{$\begin{array}{c}\text { Cristalinidade } \\
(\%)\end{array}$} & \multicolumn{2}{|c|}{$\begin{array}{c}\text { Parâmetros de } \\
\text { refinamento }\end{array}$} \\
\hline & $\begin{array}{c}(1) \\
\mathrm{NaCl}\end{array}$ & $\begin{array}{c}(2) \\
\mathrm{K}_{\mathrm{x}} \mathrm{Na}_{1-\mathrm{r}} \mathrm{Cl}\end{array}$ & $\begin{array}{c}(3) \\
\mathrm{NaK}_{3}\left(\mathrm{SO}_{4}\right)_{2}\end{array}$ & $\begin{array}{c}(4) \\
\mathrm{Mg}\left(\mathrm{SO}_{4}\right) \cdot\left(\mathrm{H}_{2} \mathrm{O}\right)\end{array}$ & $\begin{array}{c}(5) \\
\mathrm{K}_{2} \mathrm{SO}_{4}\end{array}$ & & $\mathrm{GoF}^{\mathrm{b}}$ & Rwp(\%) \\
\hline I & 94,92 & 1,48 & 1,56 & 2,04 & $\ldots$ & 95,04 & 1,71 & 28,44 \\
\hline II & 94,67 & 1,98 & 1,73 & 1,62 & $\ldots$ & 90,20 & 1,91 & 33,02 \\
\hline v & 90,54 & 2,75 & 1,11 & 3,27 & 2,33 & 93,47 & 1,76 & 35,26 \\
\hline \multirow[t]{2}{*}{$\begin{array}{c}\text { Flor de } \\
\text { sal }\end{array}$} & 98,01 & 1,39 & 0,15 & 0,45 & $\ldots$ & 94,14 & 2,42 & 21,56 \\
\hline & & & & ICSD e COD & & & & \\
\hline I & $96-900-3310$ & $96-900-3238$ & $01-074-1742$ & $01-080-0545$ & $\ldots$ & & & \\
\hline $\begin{array}{c}\text { Flor de } \\
\text { sal }\end{array}$ & $96-900-6370$ & $01-075-0303$ & $01-074-1742$ & $01-080-0545$ & $\ldots$ & & & \\
\hline \multicolumn{9}{|c|}{ "Concentração relativa das fases cristalinas. } \\
\hline${ }^{\mathrm{b}} \mathrm{GoF}=\mathrm{C}$ & dade de ajus & & & & & & & \\
\hline
\end{tabular}




\section{CONCLUSÕES}

A extração da flor de sal a partir de águas residuais de salinas, em condições controladas de umidade e temperatura, provou ser bem-sucedida. $\mathrm{O}$ aumento na densidade da solução durante a evaporação aumentou o número de sítios de nucleação, produzindo cristais de flor de sal de tamanhos menores e em maior quantidade. Foi verificado que apesar de maior quantidade de cristais, a massa total dos mesmos foi menor que aqueles formados no início da evaporação, concluindo assim que eles possuíam menor densidade. Esses cristais também apresentaram maior concentração da fase $\mathrm{Mg}\left(\mathrm{SO}_{4}\right) \cdot\left(\mathrm{H}_{2} \mathrm{O}\right)$ e o aparecimento da fase $\mathrm{K}_{2} \mathrm{SO}_{4}$. Ainda assim, foi evidente que pela técnica utilizada é possível extrair flor de sal de alta pureza, produto de elevado valor comercial, a partir de uma solução residual hipersalina, água mãe. O mecanismo de formação desses cristais sobrenadantes, denominados de flor de sal, foi atribuído à presença de bolhas como sítios nucleadores, supersaturação localizada devido ao gradiente térmico e formação de cristais cúbicos, ocos, que permitiram a flutuação do filme formado na superfície da água-mãe.

\section{AGRADECIMENTOS}

Este projeto foi financiado pelo Conselho Nacional de Desenvolvimento Científico e Tecnológico (CNPQ430863 / 2016-0) e Instituto Nacional de Engenharia de Superfícies (CNPq- 465423 / 2014-0) e Conselho Nacional de Melhoria do Ensino Superior (CAPES).

\section{BIBLIOGRAFIA}

[1] TANG, Y., YANG, R., KANG, X. "Modeling the effect of water vaporization and salt precipitation on reservoir properties due to carbon dioxide sequestration in a depleted gas reservoir," https://doi.org/10.1016/j.petlm.2017.12.003. Petroleum, v. 4, pp. 385-397, Dec. 2018.

[2] BALAREW, C. "Solubilities in seawater-type systems: Some technical and environmental friendly applications," http://citeseerx.ist.psu.edu/viewdoc/download?doi=10.1.1.608.211\&rep=rep1\&type=pdf. Pure Appl. Chem., v. 65, pp. 213-218, 1993.

[3] ROCHA, R.M., et al., "Brazilian solar saltworks - ancient uses and future possibilities," https://aquaticbiosystems.biomedcentral.com/track/pdf/10.1186/2046-9063-8-8. Aquat. Biosyst., v. 8, pp. 16, 2012.

[4] LYCHNOS, G., FLETCHER, J.P., DAVIES, P.A. "Properties of seawater bitterns with regard to liquiddesiccant cooling," https://doi.org/10.1016/j.desal.2008.11.019. Desalination, v. 250, n. 1, pp. 172-178, 2010.

[5] ALAMDARI, A., RAHIMPOUR, M.R., ESFANDIARI, N., et al., "Kinetics of magnesium hydroxide precipitation from sea bittern," https://doi.org/10.1016/j.cep.2007.02.012. Chem. Eng. Process. Process Intensif., v. 47, n. 2, pp. 215-221, 2008.

[6] HUSSEIN, A.A., ZOHDY, K., ABDELKREEM, M. "Seawater Bittern a Precursor for Magnesium Chloride Separation: Discussion and Assessment of Case Studies," https://www.researchgate.net/publication/316850869. Int. J. Waste Resour., v. 07, n. 01, pp. 1-6, 2017. [7] ARIONO, D., PURWASASMITA, M., WENTEN, I.G. "Brine effluents: Characteristics, environmental impacts, and their handling," https://www.researchgate.net/publication/310246896. J. Eng. Technol. Sci., v. 48, n. 4, pp. 367-387, 2016.

[8] LOGANATHAN, P., NAIDU, G., VIGNESWARAN, S. "Environmental Science Water Research \& Technology Mining valuable minerals from seawater : a critical," pp. 37-53, 2017.

[9] ROBERTS, D.A., JOHNSTON, E.L., KNOTT, N.A. "Impacts of desalination plant discharges on the marine environment: A critical review of published studies," Water Res., v. 44, n. 18, pp. 5117-5128, 2010.

[10] AL-BARWANI, H.H., PURNAMA, A. "Re-assessing the impact of desalination plants brine discharges on eroding beaches," https://doi.org/10.1016/j.desal.2006.03.536. Desalination, v. 204, n. 1-3 SPEC. ISS., pp. 94-101, 2007.

[11] TUREK, M. "Seawater desalination and salt production in a hybrid membrane-thermal process," https://doi.org/10.1016/S0011-9164(02)01123-2. Desalination, v. 153, n. 1-3, pp. 173-177, 2003.

[12] RAVIZKY, A., NADAV, N. "Salt production by the evaporation of SWRO brine in Eilat: a success story," https://doi.org/10.1016/j.desal.2006.03.559. Desalination, v. 205, n. 1-3, pp. 374-379, 2007.

[13] WU, L., SUN, T., HU, Y. "Simulation and optimization of salt-production process from desalination brine," https://doi.org/10.3303/CET1761287. Chem. Eng. Trans., v. 61, pp. 1735-1740, 2017.

[14] DONADIO, C., BIALECKI, A., VALLA, A., et al., Journal of Food Composition and Analysis 
"Carotenoid-derived aroma compounds detected and identified in brines and speciality sea salts (fleur de sel) produced in solar salterns," v. 24, pp. 801-810, 2011.

[15] SHAHIDZADEH, N., SCHUT, M.F.L., DESARNAUD, J., et al., "Salt stains from evaporating droplets," https://www.nature.com/articles/srep10335.pdf. Sci. Rep., v. 5, pp. 1-9, 2015.

[16] VELLA, D., MARCONE, M., DUIZER, L.M. "Physical and sensory properties of regional sea salts," https://doi.org/10.1016/j.foodres.2011.11.013. FRIN, v. 45, n. 1, pp. 415-421, 2012.

[17] DELLWIG, L.F. "Origin of the salina salt of michigan,” https://doi.org/10.1306/D4269819-2B2611D7-8648000102C1865D. J. Sediment. Petrol., v. 25, n. 2, pp. 83-110, 1955.

[18] PETTIT, D., FONTANA, P. "Characterization of sodium chloride crystals grown in microgravity," https://doi.org/10.1016/j.jcrysgro.2011.04.001. v. 324, pp. 207-211, 2011.

[19] FONTANA, P., PETTIT, D., CRISTOFORETTI, S. "Sodium chloride crystallization from thin liquid sheets, thick layers, and sessile drops in microgravity," https://doi.org/10.1016/j.jcrysgro.2015.07.026. J.

Cryst. Growth, v. 428, pp. 80-85, 2015.

[20] TAKIYAMA, H. "Morphology of $\mathrm{NaCl}$ crystals in drowning-out precipitation operation," https://doi.org/10.1205/026387698525559. Chem. Eng. Res. Des., v. 76, n. 7 A7, pp. 809-814, 1998.

[21] KANEKO, S., HIRASAWA, I. "Growth Mechanism of Hollow Sodium Chloride Single Crystals Grown from a Mixed Solvent of Cyclohexane and Acetone," https://doi.org/10.1002/ceat.201600641. Chem. Eng. Technol., v. 40, n. 7, pp. 1276-1281, 2017.

[22] VEESLER, S., PUEL, F. https://doi.org/10.1016/B978-0-444-56369-9.00021-6. Crystallization of Pharmaceutical Crystals, v. 1. 2015.

[23] MYERSON, A.S., TROUT, B.L. "Nucleation from solution," Science (80-. )., v. 341, n. 6148, pp. 855$856,2013$.

[24] DOUGHERTY, R.C. "Density of salt solutions: Effect of ions on the apparent density of water," https://doi.org/10.1021/jp010097r. J. Phys. Chem. B, v. 105, n. 19, pp. 4514-4519, 2001.

\section{ORCID}

Liliane Ferreira Araújo de Almada Vitor Rodrigo Melo de Melo Jussier de Oliveira Vitoriano Kristy Emanuel Silva Fontes Francisco Edson Nogueira Fraga Clodomiro Alves Junior https://orcid.org/0000-0003-0655-8171 https://orcid.org/0000-0001-8067-5929 https://orcid.org/0000-0002-9357-2088 https://orcid.org/0000-0001-5884-3332 https://orcid.org/0000-0003-2734-8157 https://orcid.org/0000-0002-5547-5922 